\title{
COMMUNICATION
}

\section{Prise en charge d'un patient atteint d'un fibrome ossifiant juvénile}

\author{
Lemoine S*, Guiol J**, Lenfant B*, Boedec A*, Dauzat A*, Kimakhe S*, Malthiery E*, \\ Lesclous $\mathrm{P}^{*}$ \\ * Unité Fonctionnelle de Chirurgie Orale, CHU de Nantes, 1 place Alexis Ricordeau, 44000 Nantes \\ ** Service de Chirurgie Maxillo-faciale et Stomatologie, CHU Nantes, 1 place Alexis Ricordeau, 44000 Nantes
}

\section{Introduction}

Les fibromes ossifiants (FO) sont regroupés avec les fibromes cémentifiants sous le terme de fibromes cémento-ossifiants. Ils sont rares mais représentent les tumeurs bénignes fibo-osseuses les plus fréquentes au niveau des maxillaires.

On distingue le FO conventionnel intéressant préférentiellement la femme de 30 à 40 ans au niveau mandibulaire et la forme juvénile qui intéresse les enfants et les jeunes adultes avec une agressivité locale et un taux de récidive plus important. La localisation préférentielle du FO juvénile est la région sinusienne, para sinusienne et orbitaire.

Cas clinique

Un patient de 16 ans originaire du Congo a consulté pour une tuméfaction évoluant depuis 2 ans au niveau du maxillaire droit, avec une croissance rapide. Il n'avait pas d'antécédents médicaux ni chirurgicaux notables, notamment de traumatisme facial.

A l'examen exobuccal, on notait une asymétrie faciale. L'examen endobuccal retrouvait une tuméfaction vestibulaire s'étendant des dents 13 à 16, de consistance dure, sans fluctuation. La muqueuse était d'aspect normale et la palpation indolore. Les dents en regard n'étaient pas mobiles et toutes vitales.

Par ailleurs, le patient ne présentait pas de signes d’altération de l'état général ni d'adénopathie palpable.

Les caractéristiques cliniques nous orientaient d'emblée vers une tumeur ossifiante.

Un bilan complémentaire a été réalisé avec une radiographie panoramique qui montrait une image opaque en regard des racines des dents 13, 14 et 15. Un CBCT montrait une volumineuse image dense, bien circonscrite, hétérogène, avec une zone d'opacification centrale, bien limitée et respectant la corticale.

En collaboration avec le service de chirurgie maxillo-faciale, une intervention sous anesthésie générale a été programmée. Une incision intrasulculaire avec décharge vestibulaire postérieure a permis le décollement d'un lambeau des dents 11 à 16. La tumeur apparaissait peu adhérente. Une résection à l'ostéotome et une régularisation du maxillaire à l'aide d'une fraise boule ont été réalisées. La voie d'abord a été refermée par des points séparés de fils résorbable. Un traitement antibiotique, antalgique et antiseptique local a été prescrit. Le patient a pu sortir dès le lendemain.

L'examen anatomopathologique révélait une tumeur osseuse bénigne ostéoformatrice correspondant à un FO juvénile trabéculaire.

Le suivi post opératoire immédiat était satisfaisant et un rendez-vous de contrôle clinique et radiologique a été programmé à 6 mois. 


\section{Conclusion}

La présentation clinique du FO juvénile est proche du FO conventionnel. Radiologiquement on note des calcifications intralésionnelles, histologiquement, la cellularité est plus riche.

Le pronostique du FO juvénile est bon et l'exérèse de la lésion doit être aussi complète que possible. Il n'existe pas de transformation maligne décrite dans la littérature du FO. Il est important de suivre le patient sur un long terme car la récidive du FO juvénile n’est pas négligeable (30 à 56 \%).

Discussion

Ce diagnostic est à évoquer devant toute tuméfaction osseuse du massif facial. Il est important de confronter les arguments cliniques, radiologiques et histologiques pour ne pas méconnaitre un diagnostic différentiel tel qu'un ostéosarcome, une dysplasie fibreuse ou une lésion de dysplasie cémento-osseuse.

Nom et adresse du conférencier

\section{Sarah LEMOINE}

Unité Fonctionnelle de Chirurgie Orale, CHU de Nantes

1, place Alexis Ricordeau

44000 Nantes (France)

sarah.lemoine@wanadoo.fr 